\title{
HARMONIC MOMENTS \\ OF INHOMOGENEOUS BRANCHING PROCESSES
}

\author{
DIDIER PIAU, ${ }^{*}$ Université Lyon 1
}

\begin{abstract}
We study the harmonic moments of Galton-Watson processes that are possibly inhomogeneous and have positive values. Good estimates of these are needed to compute unbiased estimators for noncanonical branching Markov processes, which occur, for instance, in the modelling of the polymerase chain reaction. By convexity, the ratio of the harmonic mean to the mean is at most 1 . We prove that, for every square-integrable branching mechanism, this ratio lies between $1-A / k$ and $1-A^{\prime} / k$ for every initial population of size $k>A$. The positive constants $A$ and $A^{\prime}$ are such that $A \geq A^{\prime}$, are explicit, and depend only on the generation-by-generation branching mechanisms. In particular, we do not use the distribution of the limit of the classical martingale associated with the Galton-Watson process. Thus, emphasis is put on nonasymptotic bounds and on the dependence of the harmonic mean upon the size of the initial population. In the Bernoulli case, which is relevant for the modelling of the polymerase chain reaction, we prove essentially optimal bounds that are valid for every initial population size $k \geq 1$. Finally, in the general case and for sufficiently large initial populations, similar techniques yield sharp estimates of the harmonic moments of higher degree.
\end{abstract}

Keywords: Branching process; harmonic moment; inhomogeneous Markov chain; polymerase chain reaction

2000 Mathematics Subject Classification: Primary 60J80

\section{Introduction and results}

We study the behaviour of the harmonic means, $1 / \mathrm{E}\left(1 / Z_{n}\right)$, of Galton-Watson processes, $\left(Z_{n}\right)_{n \geq 0}$, that are possibly inhomogeneous and have positive values. A motivation for this theoretical problem is the construction of unbiased estimators for samples of branching Markov processes when the state of an individual depends on the number of its siblings. An instance, outside the realm of pure probability, where this construction is needed arises in the modelling of the polymerase chain reaction by branching processes; see Sun (1995). In this specific case each individual has either 1 or 2 offspring, the state of the first descendant is identical to the state of its parent, and the state of the other descendant, if any, is a stochastic function of the state of its parent. We wish to estimate, for instance, the mutation rate of the reaction from a uniform sample of a given generation. Any unbiased estimator of the state of such a sample requires us to compute the harmonic-mean size of the corresponding generation. However, there exists no closed-form expression for these harmonic means, except for small initial populations and small numbers of generations. Since the mean sizes of the generations of a branching process are well known, the above problem is usually circumvented by assuming that the initial

Received 4 July 2005; revision received 13 March 2006.

* Current address: Institut Fourier UMR 5582, Université Joseph Fourier Grenoble 1, 100 rue des Maths, BP 74, 38402

Saint Martin d'Hères, France. Email address: didier.piau@ujf-grenoble.fr 
population is very large. Then an averaging effect occurs which results, roughly speaking, in the harmonic-mean size of a generation being close to its mean size.

In the context of the polymerase chain reaction, the author showed in previous papers (see Piau (2001), (2004)) that this approximation is accurate for surprisingly small initial populations, and provided sharp quantitative estimates of the discrepancy between the harmonic mean and the mean, for any initial population. These results also proved useful in establishing rigorous confidence intervals for the estimator of the mutation rate of the polymerase chain reaction; see Piau (2005). Our purpose in the present paper is to give the exact extent of this approximation phenomenon for general, possibly inhomogeneous, Galton-Watson processes with positive values. When the approximation phenomenon indeed occurs, we quantify it through nonasymptotic and essentially optimal bounds.

In what follows, $\left(Z_{n}\right)_{n \geq 0}$ denotes a positive, possibly inhomogeneous, Galton-Watson process. The distribution of this Markov process with values in $\{1,2, \ldots\}$ is characterized by a sequence, $\Xi:=\left(\xi_{n}\right)_{n \geq 1}$, of distributions on $\{1,2, \ldots\}$, as follows. For every $n \geq 1$, conditionally on the past of the process, $Z_{n}$ is the sum of $Z_{n-1}$ random variables of law $\xi_{n}$ which are independent of the past. Hence, $\xi_{n}$ denotes the law of the offspring number in the $n$th generation.

Assume that each $\xi_{n}$ is integrable and has mean $\mu_{n} \geq 1$. Then $Z_{n}$ is integrable and, if $\mathrm{E}_{k}$ denotes the expectation when $Z_{0}=k$, for any positive integer $k$, we have

$$
\mathrm{E}_{k}\left(Z_{n}\right)=k M_{n}, \quad \text { with } M_{n}:=\prod_{i=1}^{n} \mu_{i} .
$$

Furthermore, by convexity, the sequence with general term $M_{n} \mathrm{E}_{k}\left(1 / Z_{n}\right)$ is nondecreasing for $n \geq 0$. Thus, every term is at least $1 / k$. Our aim is to provide explicit bounds for the harmonic moments, implying, in particular, that $M_{n} \mathrm{E}_{k}\left(1 / Z_{n}\right)$ is close to $1 / k$ when this is so. In other words, we wish to show that the sequence with general term $M_{n} \mathrm{E}_{k}\left(1 / Z_{n}\right)$ is nearly constant. Indeed, for every fixed $n \geq 0$, as $k \rightarrow \infty$ the law of large numbers implies that $\mathrm{E}_{k}\left(1 / Z_{n}\right)$ is equivalent to $1 / k \mathrm{E}_{1}\left(Z_{n}\right)$, whose value is $1 / \mathrm{E}_{k}\left(Z_{n}\right)=1 / k M_{n}$. Much more is true, as we show below. To ease the task of the reader, we first state the consequence of our general results, in the homogeneous case.

Theorem 1. Assume that $\Xi$ is constant and square integrable. Then $\xi_{n}=\xi$ and $M_{n}=\mu^{n}$, where $\xi$ is square integrable and $\mu_{n}=: \mu \geq 1$ for every $n \geq 1$. Thus, there exists a positive constant, A, depending only on $\xi$, such that, for every integer $k>A$ and every $n \geq 0$,

$$
\frac{1}{k} \leq \mu^{n} \mathrm{E}_{k}\left(\frac{1}{Z_{n}}\right) \leq \frac{1}{k-A} .
$$

Assume further that $\mu \neq 1$. There then exists a positive constant, $A^{\prime}$, depending only on $\xi$, such that $A^{\prime} \leq A$ and, for every integer $k>A^{\prime}$,

$$
\lim _{n \rightarrow \infty} \mu^{n} \mathrm{E}_{k}\left(\frac{1}{Z_{n}}\right) \geq \frac{1}{k-A^{\prime}} .
$$

\subsection{Harmonic moments}

Theorem 1 is a consequence of a general quantitative result, stated as Theorem 2 below, which deals with inhomogeneous processes. To state and prove this result, we rely on some specific families of distributions, which we now define. 
Definition 1. For every $m \geq 1$, the generating function, $g_{m}$, of the positive, integer-valued random variable $L_{m}$ is such that, for any $t$ in $[0,1]$,

$$
\mathrm{E}\left(t^{L_{m}}\right)=: g_{m}(t)=\frac{t}{m-(m-1) t} .
$$

For any positive real number $c$, the random variable $L_{c, m}$ is such that, for any $t$ in $[0,1]$,

$$
\mathrm{E}\left(t^{L_{c, m}}\right)=: g_{c, m}(t)=g_{m}\left(t^{c}\right)^{1 / c}=\frac{t}{\left(m-(m-1) t^{c}\right)^{1 / c}} .
$$

Thus, $g_{m}=g_{1, m}$. For every $c, L_{c, m} \geq 1$ almost surely and $\mathrm{E}\left(L_{c, m}\right)=m$. If $m=1$ then $L_{m}=L_{c, m}=1$ almost surely. If $m>1$ then the distribution of $L_{m}-1$ is geometric and the distribution of $\left(L_{c, m}-1\right) / c$ is negative binomial.

Definition 2. For any positive real number $c$, let $\mathcal{A}_{c}$ denote the set of distributions of integrable random variables $L \geq 1$ such that, for any $t$ in $[0,1]$,

$$
\mathrm{E}\left(t^{L}\right) \leq g_{c, m}(t), \quad m:=\mathrm{E}(L) .
$$

In addition, let $\mathcal{A}_{c}^{\prime}$ denote the set of distributions of integrable random variables $L \geq 1$ such that, for any $t$ in $[0,1]$,

$$
\mathrm{E}\left(t^{L}\right) \geq g_{c, m}(t), \quad m:=\mathrm{E}(L) .
$$

Note that we compare the distribution of $L$ to distributions of random variables not a-priori integer valued, but with the same mean. We are now able to state our main result.

Theorem 2. (i) Let $n \geq 1$. Assume that there exists a c such that $\xi_{i}$ belongs to $\mathcal{A}_{c}$ for every $i \leq n$. Then, for every $k>c$,

$$
M_{n} \mathrm{E}_{k}\left(\frac{1}{Z_{n}}\right) \leq \frac{1}{k-c} .
$$

(ii) Assume that $M_{n} \rightarrow \infty$ as $n \rightarrow \infty$ and that there exists a c such that $\xi_{i}$ belongs to $\mathcal{A}_{c}^{\prime}$ for every $i \geq 1$. Then, for every $k>c$,

$$
\lim _{n \rightarrow \infty} M_{n} \mathrm{E}_{k}\left(\frac{1}{Z_{n}}\right) \geq \frac{1}{k-c} .
$$

Recall that, by convexity, the sequence $M_{n} \mathrm{E}_{k}\left(1 / Z_{n}\right)$ is nondecreasing. The existence of the limit as $n \rightarrow \infty$ is thus a general fact. Assertion (ii) of Theorem 2 is false if $M_{n}$ is allowed to stay bounded, or if the limit $n \rightarrow \infty$ is replaced with a finite $n$, since, for instance, the $n=0$ value is $1 / k$. On the other hand, in practical situations, the hypothesis that $M_{n} \rightarrow \infty$ is easy to check since it only involves the first moments of the generation-by-generation mechanisms.

The restriction to $k>c$ is important as well. As Proposition 1 shows, the behaviours of $\mathrm{E}_{k}\left(1 / Z_{n}\right)$ and $1 / M_{n}$ can be quite different if $k$ is not sufficiently large. Proposition 1 deals with one generation of a branching process using random variables distributed as $L_{c, m}$, as $m \rightarrow \infty$, and Corollary 1 applies this result to the $n$th generation of a branching process using random variables distributed as $L_{c, m}$ for a given $m$, as $n \rightarrow \infty$.

Definition 3. Let $Z$ denote a random variable and $\mathrm{P}_{k}^{c, m}$ a probability measure with respect to which $Z$ is distributed like the sum of $k$ independent, identically distributed copies of the random variable $L_{c, m}$. 
Proposition 1. For any $k \leq c, m \mathrm{E}_{k}^{c, m}(1 / Z) \rightarrow \infty$ as $m \rightarrow \infty$.

Corollary 1. Assume that $c$ is an integer and that $\xi_{n}$ is the distribution of $L_{c, m}$, for every $n$; hence, $M_{n}=Z_{0} m^{n}$. Then the distribution of $Z_{n}$ coincides with the distribution of the first generation of the branching process based on $L_{c, m^{n}}$. As a consequence, $m^{n} \mathrm{E}_{k}^{c, m}\left(1 / Z_{n}\right) \rightarrow \infty$ as $n \rightarrow \infty$.

Thus, when $k \leq c, \mathrm{E}_{k}\left(1 / Z_{n}\right)$ may decay on a different scale than $1 / M_{n}$; see more on this in Section 8 . However, Theorem 2 describes every square-integrable Galton-Watson process if $k$ is sufficiently large, as the following theorem shows.

Theorem 3. Any square-integrable distribution on $[1, \infty)$ belongs to $\mathcal{A}_{c}$ for sufficiently large $c$ and to $\mathcal{A}_{c}^{\prime}$ for sufficiently small $c$. Conversely, any distribution on $[1, \infty)$ which belongs to $\mathcal{A}_{c}$ is square integrable and has variance equal to at most $\mathrm{cm}(m-1)$, where $m$ denotes its mean. Likewise, the variance of any distribution on $[1, \infty)$ which belongs to $\mathcal{A}_{c}^{\prime}$ is either finite and equal to at least $\mathrm{cm}(m-1)$, or infinite.

We shall make precise the optimal values of $c$ for some common distributions.

Finally, Theorem 2 indeed describes the behaviour of $\mathrm{E}_{k}\left(1 / Z_{n}\right)$ when $k$ is sufficiently large, for any square-integrable branching process.

\subsection{The Bernoulli case}

We apply Theorem 2 to the Bernoulli case, when the number of offspring is always 1 or 2. This case is relevant in the context of the polymerase chain reaction. Our techniques yield accurate bounds of $\mathrm{E}_{k}\left(1 / Z_{n}\right)$ for every positive $k$, even $k=1$, for instance in the homogeneous case; see Theorem 5, below. We first state uniform bounds that are simple consequences of the results of Section 1.1. For every $j$, let $\delta_{j}$ denote the Dirac mass at $j$.

Theorem 4. Let $n \geq 0$. Assume that the laws, $\xi_{i}$ on $\{1,2\}$, of the offspring numbers are given by $\xi_{i}=\left(1-x_{i}\right) \delta_{1}+x_{i} \delta_{2}$, with $x_{i}$ in $[0,1]$, for every $i \leq n$. Then, for any $k \geq 2$,

$$
\frac{1}{k} \leq M_{n} \mathrm{E}_{k}\left(\frac{1}{Z_{n}}\right) \leq \frac{1}{k-1}
$$

In the homogeneous case, we can prove tighter bounds. We write $\mathrm{E}_{k}^{x}$ for $\mathrm{E}_{k}$ when $\xi_{i}=$ $(1-x) \delta_{1}+x \delta_{2}$ for every $i \geq 1$. For every $x$ in $(0,1)$, define

$$
\alpha_{1}(x):=\frac{1-x}{1+x}, \quad \alpha_{2}(x):=1-x .
$$

Then $0 \leq \alpha_{1} \leq \alpha_{2} \leq 1$ and $\alpha_{2}$ and $\alpha_{1}$ decrease from $\alpha_{2}(0)=\alpha_{1}(0)=1$ to $\alpha_{2}(1)=\alpha_{1}(1)=0$.

Theorem 5. (i) For any $k \geq 1$ and $n \geq 0$,

$$
\frac{1}{k} \leq(1+x)^{n} \mathrm{E}_{k}^{x}\left(\frac{1}{Z_{n}}\right) \leq \frac{1}{k-\alpha_{2}(x)} .
$$

(ii) For any $k \geq 1$,

$$
\lim _{n \rightarrow \infty}(1+x)^{n} \mathrm{E}_{k}^{x}\left(\frac{1}{Z_{n}}\right) \geq \frac{1}{k-\alpha_{1}(x)} .
$$


These estimates are precise enough to imply the following secondary result for $k=1$.

Proposition 2. There exists no uniform upper bound of $(1+x)^{n} \mathrm{E}_{1}^{x}\left(1 / Z_{n}\right)$ for $n \geq 0$ and $x$ in $(0,1)$, since $\lim _{x \rightarrow 0} \lim _{n \rightarrow \infty}(1+x)^{n} \mathrm{E}_{1}^{x}\left(1 / Z_{n}\right)$ is infinite. More precisely, for every $x$ in $(0,1)$ and every $n \geq 1$,

$$
\frac{c(x)}{x}<\lim _{n \rightarrow \infty}(1+x)^{n} \mathrm{E}_{1}^{x}\left(\frac{1}{Z_{n}}\right)<\frac{1}{x},
$$

where $c(x):=1-x(1-x) /(1+3 x)$ is such that $\frac{8}{9} \leq c(x)<1$.

In Theorem 5, the value of $\alpha_{1}(x)$ stems from the general construction of Section 1.1, but the value of $\alpha_{2}(x)$ does not. In other words, a direct application of Section 1.1 to the Bernoulli case yields $\alpha(x)$ instead of $\alpha_{2}(x)$, with

$$
\alpha(x):=-\frac{\log (1+x)}{\log (1-x)} .
$$

For every $x$ in $(0,1)$, we have $\alpha_{1}(x)<\alpha_{2}(x)<\alpha(x)$.

Theorem 5 follows from the more general case below.

Theorem 6. Recall that $\xi_{i}$ denotes the law of the offspring number in the ith generation, and assume that $\xi_{i}=\left(1-x_{i}\right) \delta_{1}+x_{i} \delta_{2}$ for every $i$.

(i) If $x_{i} \geq x$ for every $i \leq n$ then, for any $k \geq 1$,

$$
\frac{1}{k} \leq \mathrm{E}_{k}\left(\frac{1}{Z_{n}}\right) \prod_{i=1}^{n}\left(1+x_{i}\right) \leq \frac{1}{k-\alpha_{2}(x)} .
$$

(ii) If $x_{i} \leq x$ for every $i$ and $\sum_{i \geq 1} x_{i}$ diverges then, for any $k \geq 1$,

$$
\lim _{n \rightarrow \infty} \mathrm{E}_{k}\left(\frac{1}{Z_{n}}\right) \prod_{i=1}^{n}\left(1+x_{i}\right) \geq \frac{1}{k-\alpha_{1}(x)} .
$$

\subsection{A discontinuity result}

In the Bernoulli case, the functions $\alpha_{2}(x)$ and $\alpha_{1}(x)$ have a nonzero limit as $x \rightarrow 0^{+}$. Hence, the second part of Theorem 5 shows that the limit of the normalized harmonic moments does not always depend continuously on the parameters of the model. We show in this section that the phenomenon is general. For the sake of simplicity, we deal with the homogeneous case.

Let $\mathcal{M}$ denote a given subset of $(1, \infty)$ that has 1 as a limit point. Below, the limits as $\mu \rightarrow 1$ are implicitly restricted to values of $\mu$ in $\mathcal{M}$. For each $\mu$ in $\mathcal{M}$, let $\xi^{\mu}$ denote a distribution of mean $\mu$. If $\xi_{i}=\xi^{\mu}$ for every $i \geq 1$, define a function, $h_{k}$, on $\mathcal{M}$ by

$$
h_{k}(\mu):=\lim _{n \rightarrow \infty} \mu^{n} \mathrm{E}_{k}\left(1 / Z_{n}\right) .
$$

Proposition 3. Assume that for each $\mu$ in $\mathcal{M}$ there exist functions, $a(\mu)$ and $a^{\prime}(\mu)$, such that $\xi^{\mu}$ belongs to both $\mathcal{A}_{a(\mu)}$ and $\mathcal{A}_{a^{\prime}(\mu)}^{\prime}$. Then

$$
\frac{1}{k-a_{*}^{\prime}} \leq \liminf _{\mu \rightarrow 1} h_{k}(\mu) \leq \limsup _{\mu \rightarrow 1} h_{k}(\mu) \leq \frac{1}{k-a_{*}},
$$

where $a_{*}:=\limsup _{\mu \rightarrow 1} a(\mu)$ and $a_{*}^{\prime}:=\liminf _{\mu \rightarrow 1} a^{\prime}(\mu)$. Thus, if $a_{*}^{\prime}$ is positive then the function $h_{k}$ is not continuous at $\mu=1^{+}$. 
Theorem 7. In the homogeneous case, assume that each $\xi^{\mu}$ is the law of $1+X$, where the law of the random variable $X$ is binomial, Poisson, or geometric. Then, for every $k \geq 1, h_{k}$ is discontinuous at $\mu=1$, since $h_{k}(1)=1 / k$ and

$$
\lim _{\mu \rightarrow 1} h_{k}(\mu)=\frac{1}{k-1} .
$$

If the law of $X$ is geometric, then $h_{k}(\mu)=1 /(k-1)$ for every $\mu>1$ and $h_{k}(1)=1 / k$.

Now assume that each $\xi^{\mu}$ is the law of $L_{c, \mu}$, for a given positive integer $c$. Then $h_{k}(1)=1 / k$ for every $k$. Furthermore, for every $\mu>1, h_{k}(\mu)=1 /(k-c)$ if $k>c$ and $h_{k}(\mu)=\infty$ if $k \leq c$.

\subsection{Higher harmonic moments}

We now state an extension of Theorem 2 to higher harmonic moments. Theorem 8 is simply a special case of Proposition 11, below.

Theorem 8. (i) Let $n \geq 1$. Assume that there exists a c such that $\xi_{i}$ belongs to $\mathcal{A}_{c}$ for every $i \leq n$. Then, for every positive integer $r$ and every integer $k>r c$,

$$
M_{n}^{r} \mathrm{E}_{k}\left(\frac{1}{Z_{n}^{r}}\right) \leq \frac{1}{(k-c)(k-2 c) \cdots(k-r c)} .
$$

(ii) Assume that $M_{n} \rightarrow \infty$ as $n \rightarrow \infty$ and that there exists a c such that $\xi_{i}$ belongs to $\mathcal{A}_{c}^{\prime}$ for every $i$. Then, for every positive integer $r$ and every integer $k>r c$,

$$
\lim _{n \rightarrow \infty} M_{n}^{r} \mathrm{E}_{k}\left(\frac{1}{Z_{n}^{r}}\right) \geq \frac{1}{(k-c)(k-2 c) \cdots(k-r c)} .
$$

Corollary 2. Let $n \geq 1$. Assume that there exists a c such that $\xi_{i}$ belongs to $\mathcal{A}_{c}$ for every $i \leq n$, and write $\sigma_{k}^{2}\left(1 / Z_{n}\right)$ for the variance of $1 / Z_{n}$ when $Z_{0}=k$. Then, for every integer $k>2 c$,

$$
M_{n}^{2} \sigma_{k}^{2}\left(\frac{1}{Z_{n}}\right) \leq \frac{3 c}{k(k-c)(k-2 c)} .
$$

If, additionally, there exists a $c^{\prime}$ such that $\xi_{i}$ belongs to $\mathcal{A}_{c^{\prime}}^{\prime}$ for every $i \leq n$, then the sequence $k^{3} M_{n}^{2} \sigma_{k}^{2}\left(1 / Z_{n}\right)$ is bounded above and below by finite positive constants, independently of $n$ and $k$, for sufficiently large values of $k$.

\subsection{Related studies}

As mentioned above, Piau (2001), (2004) used preliminary versions of our results, especially in the Bernoulli case, which is relevant to the study of the polymerase chain reaction. In this specific case, we are now able to deal directly with every initial population size, even $k=1$.

Ney and Vidyashankar (2003) gave asymptotics of the harmonic moments of every integrable homogeneous branching process starting from $k=1$ particle. Furthermore, when $L \log L$ is integrable, their results specialize as follows (see also Bingham (1988) for some classical facts that are recalled below).

Let $p_{1}:=\mathrm{P}(L=1)$, let $\mu:=\mathrm{E}(L)$, and let $\gamma$ denote the Karlin-McGregor exponent of the distribution of $L$, defined by the equality $p_{1} \mu^{\gamma}=1$ ( $\gamma$ is also called the Schröder constant). Let $W$ denote the almost-sure limit of the nonnegative martingale $Z_{n} / \mu^{n}$. The Poincare function 
is the Laplace transform, $P(s):=\mathrm{E}_{1}(\exp (-s W))$, of the distribution of $W$ when $k=1$, and solves Poincaré's functional equation:

$$
P(\mu s)=f(P(s)) .
$$

Three cases may arise. First, if $r>\gamma$ then $\mathrm{E}_{1}\left(1 / Z_{n}^{r}\right) / p_{1}^{n}$ converges to a finite, positive limit whose expression is an integral which involves the Schröder function $S$, defined for any $t$ in $[0,1)$ by

$$
S(t):=\lim _{n \rightarrow \infty} \mathrm{E}_{1}\left(t^{Z_{n}}\right) / p_{1}^{n}
$$

Up to a multiplicative constant, $S$ is the unique finite solution in $[0,1)$ to Schröder's functional equation:

$$
S(f(t))=p_{1} S(t) .
$$

Second, when $r=\gamma, \mathrm{E}_{1}\left(1 / Z_{n}^{\gamma}\right) / n p_{1}^{n}$ converges to a finite, positive limit whose expression involves Poincaré and Schröder functions. Third, when $r<\gamma, \mu^{n r} \mathrm{E}_{1}\left(1 / Z_{n}^{r}\right)$ converges to a finite, positive limit. Ney and Vidyashankar (2003) provided an expression for the limit in terms of an integral of the Poincaré function. It can readily be checked that this limit is in fact $\mathrm{E}_{1}\left(1 / W^{r}\right)$, and that the limit is also an upper bound.

When $L \log L$ is not integrable the results are similar, but the normalizations $n p_{1}^{n}=n / \mu^{\gamma}$ (for $r=\gamma$ ) and $1 / \mu^{n r}$ (for $r<\gamma$ ) must be replaced by similar expressions which involve the Seneta-Heyde constants.

Restricting ourselves henceforth to the $L \log L$ case, we recall that the distribution of $W$ has a density, $w$, on the nonnegative real line such that $w(x) / x^{\gamma-1}$ is bounded to lie between two finite, positive constants as $x \rightarrow 0$; see Dubuc (1971).

The comparison of our results with those recalled above is based on two elementary lemmas.

Lemma 1. For any distribution, $\xi$, in $\mathcal{A}_{c}$, we have $\gamma(\xi) \geq 1 / c$. For any distribution, $\xi$, in $\mathcal{A}_{c}^{\prime}$, we have $\gamma(\xi) \leq 1 / c$.

In other words (see Definition 4, below),

$$
A^{\prime}(\xi) \leq 1 / \gamma(\xi) \leq A(\xi)
$$

Lemma 2. For any branching process, any $k \geq 1$, any $n \geq 0$, and any positive real number $r$, we have

$$
k^{r} \mathrm{E}_{k}\left(1 / Z_{n}^{r}\right) \leq \mathrm{E}_{1}\left(1 / Z_{n}^{r / k}\right)^{k} .
$$

Corollary 3 is not stated as such in the papers mentioned above, but it follows from results that we have recalled.

Corollary 3. For any homogeneous branching process of Schröder exponent $\gamma$ and any $k>$ $r / \gamma$, the sequence $\mu^{n r} \mathrm{E}_{k}\left(1 / Z_{n}^{r}\right)$ is bounded, as $n$ varies, by the finite constant $\mathrm{E}_{k}\left(1 / W^{r}\right)$.

An interesting feature of Corollary 3 is that it deals with the entire regime wherein such a constraint on $\mu^{n r} \mathrm{E}_{k}\left(1 / Z_{n}^{r}\right)$ may hold, that is, with every initial population size $k>r / \gamma(\xi)$. In other words, when $k \leq r / \gamma(\xi), \mu^{n r} \mathrm{E}_{k}\left(1 / Z_{n}^{r}\right)$ is not bounded. Our upper bounds are restricted to higher values of $k$, namely to the regime in which $k>r A(\xi)$.

One might think of recovering the dependence with respect to $k$ from the results of Ney and Vidyashankar (2003) even when $k \geq 2$, starting from the inequality

$$
\mathrm{E}_{k}\left(\frac{1}{W^{r}}\right) \leq \frac{\mathrm{E}_{1}\left(1 / W^{r / k}\right)^{k}}{k^{r}}
$$


However, the bounds obtained cannot be optimal for $k \geq 2$, since

$$
\mathrm{E}_{k}^{c}\left(\frac{1}{W}\right)=\frac{1}{k-c}<\frac{\mathrm{E}_{1}^{c}\left(1 / W^{1 / k}\right)^{k}}{k} .
$$

Furthermore, as stressed by Bingham (1988), the law of $W$ and, hence, the value of $\mathrm{E}\left(1 / W^{r}\right)$ may be explicitly computed only in very specific cases. In contrast with every other paper the author is aware of, the bounds provided here are explicit. The assumptions involve only elementary, step-by-step characteristics of the branching process, namely the distributions of the number of descendants at each generation. Also, we allow for inhomogeneous processes, as long as the reproduction laws all belong to a given space (either $\mathcal{A}_{c}$ or $\mathcal{A}_{c}^{\prime}$ ), and we make explicit the dependence of the bounds on the initial population.

The introduction of the family of distributions described by $g_{c, m}$, for integer values of $c$, is hardly new; see Harris (1948), for instance. A key point is that we use them for noninteger values of $c$ and as a reference scale for any square-integrable distribution. For instance, the $k=1$ Bernoulli distribution requires us to make use of values of $c$ in $(0,1)$. Although these distributions do not correspond to a branching process for noninteger values of $c$, they still satisfy a semigroup property, and this property is sufficient for our purposes. Finally, our methods do not determine the behaviour of the harmonic moments of homogeneous processes whose reproduction laws are not square integrable.

\subsection{Plan}

The remainder of the paper is organized as follows. In Section 2, we reduce the case of branching processes in $\mathcal{A}_{c}$ and $\mathcal{A}_{c}^{\prime}$ to the case of well-chosen distributions $g_{c, m}$, which we solve in Section 3. In Section 4, we show that the $\mathcal{A}_{c}$ and $\mathcal{A}_{c}^{\prime}$ cases imply the result for every square-integrable branching process. In Section 5, we deal with harmonic moments of higher degree. In Section 6, we thoroughly study the Bernoulli case, that is, the case when the offspring number is 1 or 2 , sharpening our previous results on this subject. We provide an algorithm to compute the asymptotic harmonic moments to any accuracy, and we present some simulations and conjectures about this specific case. Section 7 is a remark about sizebiased offsprings. Finally, in Section 8, we briefly explain how to deal with cases in which the asymptotic behaviours of the harmonic mean and the mean do not coincide.

\section{From $\mathscr{A}_{c}$ and $\mathscr{A}_{c}^{\prime}$ to $g_{c, m}$}

We show that every branching process whose branching mechanism uses only laws in $\mathcal{A}_{c}$ can be reduced to the case of $L_{c, m}$ for a suitable $m$, and we solve this case. Similar results hold regarding the comparison with $\mathcal{A}_{c}^{\prime}$.

\subsection{Results}

Lemma 3 describes the semigroup structure of each family $\left(g_{c, m}\right)_{m}$. This is the starting point of our computations. Corollary 5 is a special case of Corollary 4 and Corollary 4 is a consequence of Lemma 3. Corollary 4 uses Definition 3.

Lemma 3. For any positive real number $c$ and any $m \geq 1$ and $m^{\prime} \geq 1$, we have $g_{c, m} \circ g_{c, m^{\prime}}=$ $g_{c, m^{\prime \prime}}$, with $m^{\prime \prime}:=m m^{\prime}$.

Corollary 4. Let $\varphi$ denote a nonnegative, completely monotone function. For every branching process in $\mathcal{A}_{c}$, every $k \geq 1$, and every $n \geq 0$, we have

$$
\mathrm{E}_{k}\left(\varphi\left(Z_{n}\right)\right) \leq \mathrm{E}_{k}^{c, m}(\varphi(Z)), \text { where } m:=M_{n} .
$$


For every branching process in $\mathcal{A}_{c}^{\prime}$, every $k \geq 1$, and every $n \geq 0$, we have

$$
\mathrm{E}_{k}\left(\varphi\left(Z_{n}\right)\right) \geq \mathrm{E}_{k}^{c, m}(\varphi(Z)) \text {, where } m:=M_{n} .
$$

Recall that $\varphi$ is completely monotone if and only if its derivatives (the orders of which are indicated by superscripts in parentheses) are such that $(-1)^{i} \varphi^{(i)}$ is nonnegative for every positive integer $i$. Nonnegative, completely monotone functions are Laplace transforms of nonnegative measures on $[0, \infty)$; see Chapter IV of Widder (1941).

Corollary 5. For every branching process in $\mathcal{A}_{c}$ and every positive real number $r$, we have

$$
\mathrm{E}_{k}\left(1 / Z_{n}^{r}\right) \leq \mathrm{E}_{k}^{c, m}\left(1 / Z^{r}\right), \text { where } m:=M_{n} .
$$

For every branching process in $\mathcal{A}_{c}^{\prime}$ and every positive real number $r$, we have

$$
\mathrm{E}_{k}\left(1 / Z_{n}^{r}\right) \geq \mathrm{E}_{k}^{c, m}\left(1 / Z^{r}\right), \text { where } m:=M_{n} .
$$

\subsection{Proofs}

Proof of Lemma 3. Since each $g_{c, m}$ is the conjugate of $g_{m}$ by the bijection $t \mapsto t^{c}$, the case $c=1$ implies the general case. When $c=1,1 /\left(1-g_{m}(t)\right)$ is an affine function of $1 /(1-t)$. By composition, $g_{m} \circ g_{m^{\prime}}$ is also an affine function of $1 /(1-t)$ and it only remains to compute its coefficients to prove the semigroup property.

Proof of Corollary 4. The representation of completely monotone functions which we recalled after the statement of the corollary shows that

$$
\varphi(z)=\int_{0}^{1} t^{z} \mathrm{~d} \pi(t)
$$

for a given measure, $\pi$, on $[0,1]$. Thus, $\mathrm{E}_{k}\left(\varphi\left(Z_{n}\right)\right)$ is a positive, linear functional of the generating function, $\mathrm{E}_{k}\left(t^{Z_{n}}\right)$, of $Z_{n}$. The function $\mathrm{E}_{k}\left(t^{Z_{n}}\right)$ is the $k$ th power of the composition from $i=1$ to $i=n$ of the generating functions of the $\xi_{i}$. When the branching process belongs to $\mathcal{A}_{c}$, the generating function of $\xi_{i}$ is bounded above by $g_{c, \mu_{i}}$ and, thus, the composition is bounded above by the composition of the functions $g_{c, \mu_{i}}, 1 \leq i \leq n$, which equals $g_{c, m}$. Finally,

$$
\mathrm{E}_{k}\left(\varphi\left(Z_{n}\right)\right) \leq \int_{0}^{1} g_{c, m}(t)^{k} \mathrm{~d} \pi(t)=\mathrm{E}_{k}^{c, m}(\varphi(Z)) .
$$

The proof of the result for branching processes in $\mathcal{A}_{c}^{\prime}$ is similar.

Proof of Corollary 5. For every positive real number $r, \varphi(z):=1 / z^{r}$ is completely monotone. To see this, choose $\mathrm{d} \pi(t)=(\log 1 / t)^{r-1} \mathrm{~d} t / \Gamma(r) t$ in the representation of $\varphi$ which we used to prove Corollary 4.

\section{The case $g_{c, m}$}

Our task in this section is to evaluate the moments of $1 / Z$ under the measure $\mathrm{P}_{k}^{c, m}$. The cases $k>c$ and $k \leq c$ yield different asymptotic behaviours for the first moment of $1 / Z$. We begin with the direct way to deal with $\mathrm{P}_{k}^{c, m}$ when $k$ is sufficiently large, namely the computation of factorial moments of $Z$ instead of the usual moments; see Proposition 4. Starting with Lemma 4 , which gives a representation formula valid for every $k$, we study in depth the first 
harmonic moment, both in the small- $k$ and large- $k$ regimes. Corollary 5 and Lemma 5 below then imply the results of Theorem 2. Lemma 6 deals with the case $k=c$. Lemma 7 provides an alternative formulation of the integral of Lemma 4, a formulation used in Lemma 8 to treat the case $k<c$. Proposition 1 , which applies when $k \leq c$, is then an easy consequence.

\subsection{Results}

We begin with exact formulae. Theorem 8 is a consequence of Corollary 6 , below.

Proposition 4. (i) For every nonnegative integer $r$,

$$
\mathrm{E}_{k}^{c, m}(Z(Z+c) \cdots(Z+r c))=m^{r+1} k(k+c) \cdots(k+r c) .
$$

(ii) For every real number $r$ such that $k>r c$,

$$
m^{r} \mathrm{E}_{k}^{c, m}\left(\frac{\Gamma(Z / c-r)}{\Gamma(Z / c)}\right)=\frac{\Gamma(k / c-r)}{\Gamma(k / c)} .
$$

(iii) In particular, for every nonnegative integer $r$ such that $k>r c$,

$$
m^{r} \mathrm{E}_{k}^{c, m}\left(\frac{1}{(Z-c) \cdots(Z-r c)}\right)=\frac{1}{(k-c) \cdots(k-r c)} .
$$

Corollary 6. For every nonnegative integer $r$ such that $k>r c$,

$$
\frac{1}{k^{r}} \leq m^{r} \mathrm{E}_{k}^{c, m}\left(\frac{1}{Z^{r}}\right) \leq \frac{1}{(k-c) \cdots(k-r c)} .
$$

In particular, for every $k>c$,

$$
\frac{1}{k} \leq m \mathrm{E}_{k}^{c, m}\left(\frac{1}{Z}\right)<m \mathrm{E}_{k}^{c, m}\left(\frac{1}{Z-c}\right)=\frac{1}{k-c} .
$$

The following is a slight generalization of the assertion for $r=1$ in Corollary 6 .

Proposition 5. For every $c>0$, every $m \geq 1$, every $u \geq 0$, and every positive integer $k>u$,

$$
\frac{1}{k} \leq m \mathrm{E}_{k}^{c, m}\left(\frac{1}{Z-u}\right) \leq \frac{1}{k-\sup \{c, u\}} .
$$

Proposition 4 and Corollary 6 are the results that we use to treat the case $k>c$ in the rest of the paper. We now turn to the evaluation of the exact harmonic moment of $Z$ with respect to $\mathrm{P}_{k}^{c, m}$. The results below are mostly used to deal with the case $k \leq c$.

Lemma 4. For every positive integer $k$, every positive real number $c$, and every $m>1$,

$$
\mathrm{E}_{k}^{c, m}\left(\frac{1}{Z}\right)=\frac{G(k / c, m)}{c}, \quad G(u, m):=\int_{0}^{1} \frac{t^{u-1} \mathrm{~d} t}{1+(m-1) t} .
$$

Alternatively,

$$
G(u, m)=\frac{B_{u, 1-u}(1-1 / m)}{(m-1)^{u}},
$$

where $B_{u, v}$ denotes the incomplete beta function with parameters $u$ and $v$, that is, for every $x$ in $[0,1)$,

$$
B_{u, v}(x):=\int_{0}^{x} t^{u-1}(1-t)^{v-1} \mathrm{~d} t .
$$


Lemma 5. Assume that $u>1$. Then

$$
m G(u, m) \leq \frac{1}{u-1} .
$$

The order of this upper bound is exact when $m$ is large, since the function $(m-1) G(u, m)$ increases as $m$ increases and converges to $1 /(u-1)$ as $m \rightarrow \infty$.

Lemma 6. $G(1, m)=(\log m) /(m-1)$.

Lemma 7. For any $u,(m-1)^{u} G(u, m)$ is an increasing function of $m \geq 1$.

Lemma 8. Assume that $u<1$. As $m \rightarrow \infty,(m-1)^{u} G(u, m)$ converges to $c_{u}:=\pi / \sin (\pi u)$. Thus, on the one hand, for any $m>1,(m-1)^{u} G(u, m) \leq c_{u}$. On the other hand, for any $m \geq 2,(m-1)^{u} G(u, m) \geq 1 / 2 u$. For every $u<1$, bounds of $c_{u}$ are $c_{u} \geq \pi$ and

$$
\frac{1}{2 u(1-u)} \leq c_{u} \leq \frac{1}{u(1-u)}
$$

Corollary 7. Let $k<c$ and $\ell(k, c):=c / k(c-k)$. For every $m$,

$$
(m-1)^{k / c} \mathrm{E}_{k}^{c, m}(1 / Z) \leq \ell(k, c) .
$$

The order of this upper bound is exact, since

$$
\lim _{m \rightarrow \infty}(m-1)^{k / c} \mathrm{E}_{k}^{c, m}(1 / Z) \geq \frac{1}{2} \ell(k, c) .
$$

\subsection{Proofs}

Lemmas 6, 7, and 8 follow from the definitions.

Proof of Proposition 4. (i) For any $x$ with $|x|<1$ and any positive real number $y$,

$$
\frac{1}{(1-x)^{y}}=\sum_{r \geq 0} x^{r} \frac{\Gamma(y+r)}{\Gamma(y) \Gamma(r+1)} .
$$

Setting $y=Z / c$ and integrating yields

$$
\mathrm{E}_{k}^{c, m}\left(\frac{1}{(1-x)^{Z / c}}\right)=\sum_{r \geq 0} \mathrm{E}_{k}^{c, m}\left(\frac{\Gamma(r+Z / c)}{\Gamma(Z / c)}\right) \frac{x^{r}}{\Gamma(r+1)} .
$$

However, we also have

$$
\mathrm{E}_{k}^{c, m}\left(\frac{1}{(1-x)^{Z / c}}\right)=g_{c, m}\left(\frac{1}{(1-x)^{1 / c}}\right)^{k}=\frac{1}{(1-m x)^{k / c}} .
$$

Using the expansion of $1 /(1-m x)^{k / c}$ given above and equating the coefficients of the two series yields the result for any nonnegative integer $r$.

(ii) For any positive real numbers $y$ and $r$ with $y>r$,

$$
\frac{\Gamma(r) \Gamma(y-r)}{\Gamma(y)}=\int_{0}^{1} t^{y-r-1}(1-t)^{r-1} \mathrm{~d} t .
$$


Setting $y=Z / c$ and performing the integration yields

$$
\Gamma(r) \mathrm{E}_{k}^{c, m}\left(\frac{\Gamma(Z / c-r)}{\Gamma(Z / c)}\right)=\int_{0}^{1} g_{c, m}\left(t^{1 / c}\right)^{k} \frac{(1-t)^{r-1}}{t^{r+1}} \mathrm{~d} t .
$$

The change of variable $s:=g_{c, m}\left(t^{1 / c}\right)^{c}=g_{m}(t)$ yields

$$
\Gamma(r) \mathrm{E}_{k}^{c, m}\left(\frac{\Gamma(Z / c-r)}{\Gamma(Z / c)}\right)=\int_{0}^{1} s^{k / c-r-1} \frac{(1-s)^{r-1}}{m^{r}} \mathrm{~d} s,
$$

which is the desired formula.

Proof of Lemma 4. Write $\mathrm{E}_{k}^{c, m}(1 / Z)$ as the integral of $g_{c, m}(t)^{k} / t$ over $(0,1)$. Use the change of variable $t^{\prime}:=g_{c, m}(t)^{c}$. This yields the first expression for $G$ in the lemma. To obtain the expression for $G$ in terms of the incomplete beta function, use the change of variable $t^{\prime}:=(m-1) t /(1+(m-1) t)$ in the first expression for $G$.

Proof of Lemma 5. In the first expression for $G$ in Lemma 4, use the fact that $1+(m-1) t$ lies between $m t$ and $m$. Thus, $G(u, m)$ lies between the integral of $t^{u-2} / m$ and the integral of $t^{u-1} / m$, that is, between $1 /(u-1) m$ and $1 / u m$.

Proof of Corollary 7. Lemma 4 and Lemma 8 imply that the left-hand side of the inequality is indeed bounded by $c_{u} / c$, where $u:=k / c$. Therefore, we can use the bound of $c_{u}$ by $1 / u(1-u)$ in Lemma 8 . This yields the bound for every finite value of $m$. The limit as $m \rightarrow \infty$ is $c_{u} / c \geq 1 / 2 u c(1-u)=\ell(k, c) / 2$.

\section{From $\mathcal{A}_{c}$ and $\mathcal{A}_{c}^{\prime}$ to the general case}

In this section, we show that every square-integrable branching process belongs either to the set $\mathcal{A}_{c}$ or to the set $\mathcal{A}_{c}^{\prime}$, for a suitable value of $c$; we prove Theorem 3; and we describe the best possible values for the constants, $c$, of Theorem 2 in some specific examples.

\subsection{Comparisons}

Our next proposition is related to Theorem 3 and motivates Definition 4, below.

Proposition 6. If $c_{1} \leq c_{2}$ and $m \geq 1$ then $g_{c_{1}, m} \leq g_{c_{2}, m}$. If $c_{1}<c_{2}$ and $m>1$ then the distribution of $L_{c_{2}, m}$ belongs to $\mathcal{A}_{c_{2}}$ but not to $\mathcal{A}_{c_{1}}$ and the distribution of $L_{c_{1}, m}$ belongs to $\mathcal{A}_{c_{1}}^{\prime}$ but not to $\mathcal{A}_{c_{2}}^{\prime}$. Thus, $\left(\mathcal{A}_{c}\right)_{c}$ is a strictly increasing sequence and $\left(\mathcal{A}_{c}^{\prime}\right)_{c}$ is a strictly decreasing sequence.

Definition 4. For any square-integrable distribution $\xi$ on $[1, \infty)$, let

$$
A(\xi):=\inf \left\{c>0: \xi \in \mathcal{A}_{c}\right\}, \quad A^{\prime}(\xi):=\sup \left\{c>0: \xi \in \mathcal{A}_{c}^{\prime}\right\} .
$$

\subsection{Examples}

We now study some specific transformations and examples. Proposition 7 follows from the definitions.

Proposition 7. For every $\xi$, we have $A^{\prime}(\xi) \leq A(\xi)$. The inequality is strict except in two cases: when $A(\xi)=A^{\prime}(\xi)=0$, in which case $\xi$ is a Dirac measure at $m \geq 1$, and when $A(\xi)=A^{\prime}(\xi)=c$ is positive, in which case $\xi$ is the distribution of a random variable $L_{c, m}$. 
Proposition 8. (i) If the laws of the independent random variables $1+X$ and $1+X^{\prime}$ belong to $\mathcal{A}_{c}^{\prime}$, then the law of $1+X+X^{\prime}$ also belongs to $\mathcal{A}_{c}^{\prime}$. This statement is false if $\mathcal{A}_{c}^{\prime}$ is replaced by $\mathcal{A}_{c}$.

(ii) If the law of $1+X$ belongs to $\mathcal{A}_{c}$ and $b$ is positive, then the law of $1+b X$ belongs to $\mathcal{A}_{c b}$. A similar statement holds if $\mathcal{A}_{c}$ and $\mathcal{A}_{c b}$ are respectively replaced by $\mathcal{A}_{c}^{\prime}$ and $\mathcal{A}_{c b}^{\prime}$.

(iii) If the law of $L$ belongs to $\mathcal{A}_{c}$ and $L^{\prime}$ stochastically dominates $L$, then the law of $L^{\prime}$ also belongs to $\mathcal{A}_{c}$. For instance, if $b$ is nonnegative then the law of $L+b$ belongs to $\mathcal{A}_{c}$. A similar statement holds if $\mathcal{A}_{c}$ is replaced by $\mathcal{A}_{c}^{\prime}$.

If $\xi$ is a Dirac measure then $A^{\prime}(\xi)=A(\xi)=0$. Other common cases are as follows.

Proposition 9. (i) If $\xi$ is uniform on $\{1, \ldots, n\}$ then $A(\xi)<1$; more precisely, $2 n^{A(\xi)}=n+1$.

(ii) If $\xi$ is uniform on $\{1, n\}$ then $A^{\prime}(\xi)=(n-1) /(n+1)$ and $2^{A(\xi)+1}=n+1$.

(iii) If $\xi$ is the law of $1+X$, where $X$ is $\operatorname{binomial}(n, x)$-distributed, then $A(\xi)<1$; more precisely, $(1+x n)(1-x)^{n A(\xi)}=1$.

(iv) If $\xi$ is the law of $1+X$, where $X$ is Poisson-distributed with mean $x$, then $A(\xi)<1$; more precisely, $\mathrm{e}^{x A(\xi)}=1+x$.

In the notation of Section 1.5, cases (i) to (iv) of Proposition 9 are such that $A(\xi)=1 / \gamma(\xi)>$ $A^{\prime}(\xi)$. To check that the three values $A(\xi), 1 / \gamma(\xi)$, and $A^{\prime}(\xi)$ can indeed be different, assume that $\xi:=(1-p) \delta_{1}+\left(\delta_{2}+\delta_{3}\right) p / 2$ with $p \in(0,1)$. Then $\gamma(\xi):=-\log (1-p) / \log (1+3 p)$. For $p=\frac{1}{5}$, we can check that the function $t \mapsto \mathrm{E}\left(t^{L}\right) / g_{1 / \gamma, m}(t)$ has positive derivative at $t=0$ and $t=1$. Thus, some values of this function are greater than 1 and some are smaller than 1. This implies that $A(\xi)>1 / \gamma(\xi)>A^{\prime}(\xi)$.

\subsection{The Bernoulli case}

Definition 5. If $\xi=(1-x) \delta_{1}+x \delta_{2}$ then we write $\alpha(x)$ for $A(\xi)$ and $\alpha_{1}(x)$ for $A^{\prime}(\xi)$.

Proposition 10. For any $x$ in $(0,1)$, we have $\alpha_{1}(x)<\alpha(x)<1$, since

$$
\alpha_{1}(x)=\frac{1-x}{1+x}, \quad(1-x)^{\alpha(x)}(1+x)=1 .
$$

Thus, $\alpha$ and $\alpha_{1}$ decrease in $(0,1]$, from $\alpha\left(0^{+}\right)=\alpha_{1}\left(0^{+}\right)=1$ to $\alpha(1)=\alpha_{1}(1)=0$. Both are discontinuous at 0 , since $\alpha(0)=\alpha_{1}(0)=0$.

Note that $\alpha_{1}(x) \leq 1-x \leq \alpha(x)$.

\subsection{Proofs}

Proof of Proposition 6. To prove this result, simply compare the logarithmic derivatives.

Proof of Theorem 3. Both results stem from the expansion of $g_{c, m}$ near 1, which reads as follows: when $t=o(1)$,

$$
g_{c, m}(1-t)=1-m t+\frac{1}{2} c(c+1) m(m-1) t^{2}+o\left(t^{2}\right) .
$$

We also have

$$
\mathrm{E}\left((1-t)^{L}\right)=1-\mathrm{E}(L) t+\frac{1}{2} \mathrm{E}(L(L-1)) t^{2}+o\left(t^{2}\right)
$$


A comparison of the second-order terms of these expansions yields the conditions on the variance of $L$ for $L$ to belong to $\mathcal{A}_{c}$ and, respectively, $\mathcal{A}_{c}^{\prime}$.

To show that any square-integrable distribution belongs to $\mathcal{A}_{c}$ for suitable values of $c$, we first choose values of $d$ and $s<1$ sufficiently large to ensure that $\mathrm{E}\left(t^{L}\right) \leq g_{d, m}(t)$ for every $t \geq s$. According to the expansion above, this is possible for any $d$ such that

$$
d(d+1) m(m-1)>\mathrm{E}(L(L-1)), \quad m:=\mathrm{E}(L) .
$$

We then choose a value of $c>d$ sufficiently large that $1 / m^{1 / c} \geq g_{d, m}(s) / s$. Thus, $\mathrm{E}\left(t^{L}\right) \leq$ $g_{d, m}(t) \leq g_{c, m}(t)$ for every $t \geq s$ and, since $\mathrm{E}\left(t^{L}\right) / t$ is a nondecreasing function of $t$,

$$
\mathrm{E}\left(t^{L}\right) \leq t \mathrm{E}\left(s^{L}\right) / s \leq t g_{d, m}(s) / s \leq t / m^{1 / c} \leq g_{c, m}(t)
$$

for any $t \leq s$. The proof for the comparison with distributions in $\mathcal{A}_{c}^{\prime}$ is similar.

Proof of Proposition 8. Part (i) follows from the fact that

$$
g_{c, m}(t) g_{c, m^{\prime}}(t) \geq t g_{c, m m^{\prime}}(t) .
$$

We leave the verification of this as an exercice for the reader. Parts (ii) and (iii) are clear, and their proofs thus omitted.

\section{Higher moments}

Assume that $\xi_{i}$ belongs to $\mathcal{A}_{c}$ for every $i \leq n$, and let $m:=M_{n}$. Then

$$
M_{n}^{r} \mathrm{E}_{k}\left(1 / Z_{n}^{r}\right) \leq m^{r} \mathrm{E}_{k}^{c, m}\left(1 / Z^{r}\right) .
$$

Expansions of $g_{c, m}(t)$ in the limit as $m \rightarrow \infty$ show that the distribution of $Z / m$ with respect to $\mathrm{P}_{1}^{c, m}$ converges to the distribution of $W$ with respect to a measure, $\mathrm{P}_{1}^{c}$, such that

$$
\mathrm{E}_{1}^{c}\left(\mathrm{e}^{-t W}\right)=(1+c t)^{-1 / c} .
$$

The distribution of $W$ is gamma $(c, 1 / c)$, that is, its density with respect to the Lebesgue measure, $\mathrm{d} w$, is

$$
w^{c-1} \mathrm{e}^{-w / c} \mathbf{1}_{\{w \geq 0\}} / c^{c} \Gamma(c) .
$$

Furthermore, $g_{c, m}(t) \leq g_{c}(t):=\mathrm{E}_{1}^{c}\left(t^{W}\right)=(1-c \log t)^{-1 / c}$. Hence,

$$
M_{n}^{r} \mathrm{E}_{k}\left(\frac{1}{Z_{n}^{r}}\right) \leq \mathrm{E}_{k}^{c}\left(\frac{1}{W^{r}}\right)=\frac{\Gamma(k / c-r)}{c^{r} \Gamma(k / c)} .
$$

This inequality holds for every positive value of $r$ and $c$ and every positive integer $k$ such that $k>c r$. These results prove the following proposition.

Proposition 11. (i) Consider a $c$ such that $\xi_{i}$ belongs to $\mathscr{A}_{c}$ for every $i \leq n$. For every positive real number $r$ and every $k$ such that $k>r c$, we have

$$
M_{n}^{r} \mathrm{E}_{k}\left(\frac{1}{Z_{n}^{r}}\right) \leq \frac{1}{[k, c]_{r}}, \quad \text { where }[k, c]_{r}:=\frac{c^{r} \Gamma(k / c)}{\Gamma(k / c-r)} .
$$

When $r$ is an integer, $[k, c]_{r}=\prod_{i=1}^{r}(k-i c)$.

(ii) Conversely, consider a $c$ such that $\xi_{i}$ belongs to $\mathcal{A}_{c}^{\prime}$ for every $i$. Assume that $M_{n} \rightarrow \infty$. Then, for every $k$ and every positive real number $r \geq k / c$,

$$
\lim _{n \rightarrow \infty} M_{n}^{r} \mathrm{E}_{k}\left(1 / Z_{n}^{r}\right)=\infty
$$




\section{Bernoulli branching processes}

\subsection{Preliminaries}

We first set some notation, to be able to deal with inhomogeneous processes.

Definition 6. The efficiency of a Bernoulli branching process is the sequence $\mathcal{X}:=\left(x_{i}\right)_{i \geq 1}$ such that $\xi_{i}=\left(1-x_{i}\right) \delta_{1}+x_{i} \delta_{2}$. Let $\mathbb{L}$ and $\mathbb{L}^{*}$ denote the sets of efficiencies such that $x_{i}$ belongs to $[0,1]$ and, respectively, $(0,1]$ for every $i \geq 1$. For any $\mathcal{X}$ in $\mathbb{L}$, let $s(\mathcal{X}):=\left(x_{i+1}\right)_{i \geq 1}$ denote the shifted sequence.

Definition 7. For any $k \geq 1$ and any efficiency $\mathcal{X}$, let

$$
B_{k}(X):=\lim _{n \rightarrow \infty} \mathrm{E}_{k}\left(1 / Z_{n}\right) \prod_{i=1}^{n}\left(1+x_{i}\right) .
$$

In the homogeneous case, in which $x_{i}=x$ for every $i \geq 1$, we write $B_{k}(x)$ for $B_{k}(\mathcal{X})$.

By convexity, the limit which defines $B_{k}(\mathcal{X})$ is also a supremum over $n \geq 0$; thus, $B_{k}(\mathcal{X}) \geq$ $1 / k$. The functional $B_{k}$ also describes $\mathrm{E}_{k}\left(1 / Z_{n}\right)$ for finite values of $n$, since replacing every $x_{i}, i \geq n+1$, by 0 freezes the branching process at the value $Z_{n}$. Thus, uniform upper bounds of $B_{k}$ on $\mathbb{L}$ yield upper bounds of $\mathrm{E}_{k}\left(1 / Z_{n}\right)$ for finite values of $n$.

\subsection{Results}

The following result holds uniformly over the set $\mathbb{L}$ and is a consequence of the fact that $A(\xi)<1$ for every Bernoulli distribution $\xi$; see Proposition 10.

Proposition 12. For every efficiency $\mathcal{X}$ in $\mathbb{L}$ and every $k \geq 1$,

$$
\frac{1}{k} \leq B_{k}(X) \leq \frac{1}{k-1}
$$

Thus, for every $n \geq 0$,

$$
\frac{1}{k} \leq \mathrm{E}_{k}\left(\frac{1}{Z_{n}}\right) \prod_{i=1}^{n}\left(1+x_{i}\right) \leq \frac{1}{k-1} .
$$

The sequence $\left(B_{k}\right)_{k \geq 1}$ satisfies recursion relations, which we state in Proposition 13, that characterize it fully; see Proposition 14.

Proposition 13. For every $k \geq 1$, the function $B_{k}$ is measurable on $\mathbb{L}$. Furthermore, for every $k \geq 1$ and $\mathcal{X}$ in $\mathbb{L}$,

$$
B_{k}(\mathcal{X})=\left(1+x_{1}\right) \sum_{i}\left(\begin{array}{l}
k \\
i
\end{array}\right) x_{1}^{i}\left(1-x_{1}\right)^{k-i} B_{k+i}(s(\mathcal{X})) .
$$

Proposition 14. Let $\left(F_{k}\right)_{k \geq 1}$ denote a sequence of functionals defined on $\mathbb{L}^{*}$. Assume that, as $k \rightarrow \infty, k F_{k}(X) \rightarrow 1$ uniformly over $\mathcal{X}$ in $\mathbb{L}^{*}$, and that $\left(F_{k}\right)_{k \geq 1}$ solves (1) on $\mathbb{L}^{*}$ for every $k \geq 1$. Then $F_{k}=B_{k}$ on $\mathbb{L}^{*}$ for every $k \geq 1$.

The sequence $\left(B_{k}\right)_{k \geq 1}$ is entirely determined on $\mathbb{L}^{*}$ by the recursion (1) and by the bounds $1 / k \leq B_{k} \leq 1 /(k-1)$. 
Note finally that the recursion (1) is just a special case of the following result. For any branching process of reproduction law $\Xi=\left(\xi_{i}\right)_{i \geq 1}$ and any $k \geq 1$, introduce

$$
H_{k}(\Xi):=\lim _{n \rightarrow \infty} M_{n} \mathrm{E}_{k}\left(1 / Z_{n}\right)
$$

and the shifted mechanism $s(\Xi):=\left(\xi_{i+1}\right)_{i \geq 1}$. Let $\xi_{1}^{* k}$ denote the $k$-fold convolution of the measure $\xi_{1}$ with itself. Then

$$
H_{k}(\Xi)=\mu_{1} \sum_{i \geq k} \xi_{1}^{* k}(i) H_{i}(s(\Xi))
$$

\subsection{The homogeneous case}

We start with a version of the relation (1) in the homogeneous case.

Proposition 15. For every $x$ in $(0,1)$,

$$
B_{k}(x)=(1+x) \sum_{i}\left(\begin{array}{l}
k \\
i
\end{array}\right) x^{i}(1-x)^{k-i} B_{k+i}(x) .
$$

The recursion whose left-hand side is $B_{k}(x)$ involves the whole set of values $B_{k}(x), B_{k+1}(x)$, $\ldots, B_{2 k}(x)$. Thus, this system of equations does not directly yield the value of each $B_{k}(x)$. The exception is the case $k=1$.

Corollary 8. For every $x \neq 0, B_{1}(x)=B_{2}(x)(1+x) / x$.

Our main result in this section is Proposition 16.

Proposition 16. Let $\alpha_{1}(x):=(1-x) /(1+x)$ and $\alpha_{2}(x):=1-x$. For every $k \geq 1$,

$$
\frac{1}{k-\alpha_{1}(x)} \leq B_{k}(x) \leq \frac{1}{k-\alpha_{2}(x)} .
$$

Thus, $B_{k}\left(0^{+}\right)=1 /(k-1)$ and $B_{k}\left(1^{-}\right)=1 / k$.

For $k=1$, Proposition 16 states that $B_{1}(x)$ is at least $1 /\left(1-\alpha^{\prime}(x)\right)$. A tighter bound, namely

$$
\frac{(1+x)^{2}}{1+3 x} \leq x B_{1}(x) \leq 1,
$$

obtains if we use Corollary 8 first and then Proposition 16. The lower bound is always greater than $\frac{8}{9}=0.8889^{-}$. In our numerical simulations in Section 6.9, below, some values of $\lambda B_{1}(\lambda)$ are found to be as small as $B_{*}=0.9274 \pm 0.0002$.

We could iterate the procedure, obtaining yet tighter upper and lower bounds for $\mathrm{E}_{1}^{x}\left(1 / Z_{n}\right)$ or for any $\mathrm{E}_{k}^{x}\left(1 / Z_{n}\right)$ with $k \geq 1$, to any prescribed accuracy. We develop this idea in Section 6.7.

We end this section with a conjecture.

Conjecture 1. Every function $x \mapsto B_{k}(x)$ is decreasing in $x$ on $(0,1]$.

A problem for further research is to prove this and find a natural explanation of the fact that $B_{k}\left(0^{+}\right)$and $B_{k}(0)$ are not equal. 


\subsection{Proofs}

Proof of Proposition 13. Since each $\mathrm{E}_{k}\left(1 / Z_{n}\right)$ is measurable with respect to $\left(x_{i}\right)_{i \leq n}, B_{k}(x)$ is the limit of a measurable, nondecreasing sequence; hence, $B_{k}$ is measurable. Regarding the recursion relation, we consider the conditioning on $Z_{1}$ of the Bernoulli branching process starting from $Z_{0}=k$. On the event $\left\{Z_{1}=k+i\right\},\left(Z_{n+1}\right)_{n \geq 0}$ follows the law of a GaltonWatson branching process of efficiency $s(x)$ starting from $k+i$. Hence, (1) follows from the fact that the distribution of $Z_{1}-k$ is $\operatorname{binomial}\left(k, x_{1}\right)$.

Proof of Proposition 14. We show that the conditions in the statement of Proposition 14 define $\left(B_{k}\right)_{k}$ uniquely. The existence follows from the construction of each $B_{k}$. A proof of the uniqueness is as follows. Assume that the sequences of functionals $\left(B_{k}^{\prime}\right)$ and $\left(B_{k}^{\prime \prime}\right)$ are solutions. In particular, $B_{k}^{\prime} / B_{k}^{\prime \prime} \rightarrow 1$ uniformly on $\mathbb{L}^{*}$ as $k \rightarrow \infty$. Fix an $\varepsilon$. For every sufficiently large $k$ and every $x$ in $\mathbb{L}^{*}$,

$$
B_{k}^{\prime}(x) \leq(1+\varepsilon) B_{k}^{\prime \prime}(x) .
$$

Since $\left(B_{k}^{\prime}\right)$ and $\left(B_{k}^{\prime \prime}\right)$ both solve the recursion relation (1), a recursion over the decreasing values of $k$ shows that $B_{k}^{\prime}(x) \leq(1+\varepsilon) B_{k}^{\prime \prime}(x)$ for every $k \geq 1$ and every $x$ in $\mathbb{L}^{*}$. This recursion uses as a crucial tool the fact that no $x_{k}$ is 0 . Now, since $\varepsilon$ is arbitrary, we have $B_{k}^{\prime} \leq B_{k}^{\prime \prime}$ on $\mathbb{L}^{*}$ for every $k \geq 1$. By exchanging the roles of the two sequences, we see that $B_{k}^{\prime}=B_{k}^{\prime \prime}$ on $\mathbb{L}^{*}$ for every $k \geq 1$.

\subsection{Outline of the proof of Proposition 16}

We start from relations between the functions $B_{k}$ in Proposition 15, which read

$$
B_{k}(x)=(1+x) \mathrm{E}_{k}^{x}\left(B_{Z_{1}}(x)\right), \quad k \geq 1 .
$$

With respect to the probability $\mathrm{P}_{k}^{x}, Z_{1}$ is distributed like the sum of $k$ independent, identically distributed random variables of distribution $(1-x) \delta_{1}+x \delta_{2}$. The next lemma follows from the fact that $k B_{k}(x) \rightarrow 1$ as $k \rightarrow \infty$.

Lemma 9. Assume that $\lim _{\inf _{k \rightarrow \infty}} k \varphi(k) \geq 1$ and that, for every $k \geq 1$,

$$
(1+x) \mathrm{E}_{k}^{x}\left(\varphi\left(Z_{1}\right)\right) \leq \varphi(k) .
$$

Then $B_{k}(x) \leq \varphi(k)$ for every $k \geq 1$. Conversely, if $\limsup _{k \rightarrow \infty} k \psi(k) \leq 1$ and, for every $k \geq 1$,

$$
(1+x) \mathrm{E}_{k}^{x}\left(\psi\left(Z_{1}\right)\right) \geq \psi(k)
$$

then $B_{k}(x) \geq \psi(k)$ for every $k \geq 1$.

Definition 8. For every $k \geq 1$, let $c_{k}$ denote the unique solution in $(0,1)$ to the equation

$$
(1+x) \mathrm{E}_{k}^{x}\left(\frac{1}{Z_{1}-c_{k}}\right)=\frac{1}{k-c_{k}} .
$$

The next lemma becomes obvious if an equivalent definition of $c_{k}$, given below in part (ii) of Lemma 13, is used.

Lemma 10. If $c_{k} \leq c$ for every $k \geq 1$ then $B_{k}(x) \leq 1 /(k-c)$ for every $k \geq 1$. Conversely, if $c_{k} \geq c$ for every $k \geq 1$ then $B_{k}(x) \geq 1 /(k-c)$ for every $k \geq 1$.

Lemma 10 asserts that $B_{k}(x) \leq 1 /(k-c)$ for $c=\sup \left\{c_{k}: k \geq 1\right\}$ and, by Lemma 11, this supremum is $c_{1}=1-x$; Proposition 16 thus follows. 
Lemma 11. For every $k \geq 1, c_{k} \leq c_{1}=1-x$.

The following result shows that the technique above cannot yield a better value of $\alpha_{1}(x)$ than $\alpha_{1}(x)=(1-x) /(1+x)$.

Lemma 12. As $k \rightarrow \infty, c_{k} \rightarrow(1-x) /(1+x)$. Furthermore, for $k \geq 2, c_{k}+x c_{k-1} \geq 1-x$.

Finally, we use the characterizations below to evaluate $c_{k}$.

Lemma 13. For every $k \geq 2$, the following inequalities are equivalent to each other and to the fact that $c \geq c_{k}$ :

(i) $(1+x) \mathrm{E}_{k}^{x}\left(1 /\left(Z_{1}-c\right)\right) \leq 1 /(k-c)$,

(ii) $k(1+x) \mathrm{E}_{k-1}^{x}\left(1 /\left(Z_{1}+2-c\right)\right) \geq 1$,

(iii) $k(k-1) x(1+x) \mathrm{E}_{k-2}^{x}\left(1 /\left(Z_{1}+4-c\right)\right) \leq x k-1+c$.

The reversed inequalities, $\left(i^{\prime}\right),\left(i i^{\prime}\right)$, and (iii'), are equivalent to each other and to the fact that $c \leq c_{k}$.

\subsection{Technical steps of the proof of Proposition 16}

We prove Lemmas 11 and 12, assuming Lemma 13 to hold for the moment. By Jensen's inequality, the expectation of the inverse is greater than the inverse of the expectation. Thus, inequality (ii') of Lemma 13 implies that

$$
k(1+x) \leq(k-1)(1+x)+2-c .
$$

This reads $c \leq 1-x$. Since $c_{1}=1-x$, Lemma 11 follows. Furthermore, we can and do restrict to $c \leq 1-x$ in the reasoning below.

To prove Lemma 12, we first note that inequality (ii) involves the expected value of a concave function of $u:=1 /\left(Z_{1}-c_{k-1}\right)$, namely the function $u \mapsto u /(1+b u)$ with $b:=c_{k-1}+2-c$. The expected value of a concave function is at most the value of the function at the expected value of its argument. From the definition of $c_{k-1}$, inequality (ii) implies that

$$
k(1+x) \geq\left(k-1-c_{k-1}\right)(1+x)+c_{k-1}+2-c .
$$

This is equivalent to $c \geq 1-x-x c_{k-1}$. Hence, for any $k \geq 2$,

$$
1-x-x c_{k-1} \leq c_{k} \leq 1-x .
$$

This is enough to show that $c_{k} \geq(1-x)^{2}$ for every $k \geq 1$. Thus, we can and do further restrict to $c \geq(1-x)^{2}$ in the reasoning below.

In the second step of the proof of Lemma 12, we use inequality (iii') in the way we used inequality (ii). That is, we note that inequality (iii') involves the expected value of a concave function of $1 /\left(Z_{1}-c_{k-2}\right)$ and apply Jensen's inequality once again. From the definition of $c_{k-2}$, inequality (iii') implies that

$$
k(k-1) x(1+x) \geq\left[(1+x)\left(k-2-c_{k-2}\right)+c_{k-2}+4-c\right](x k-1+c) .
$$

After some simplifications, this reads $A_{1} k+A_{0} \geq 0$, with

$$
A_{1}:=(1-x)^{2}-c+x^{2} c_{k-2}, \quad A_{0}:=(1-c)\left(2(1-x)-c-x c_{k-2}\right) .
$$


Since $c \geq(1-x)^{2}$ and $c_{k-2} \geq(1-x)^{2}$, simple bounds show that $A_{0} \leq 1$. Hence, inequality (iii') implies that $A_{1} \geq-1 / k$. Finally, for every $k \geq 3$, we have

$$
(1-x)^{2} \leq c_{k} \leq(1-x)^{2}+x^{2} c_{k-2}+1 / k .
$$

We use the a-priori bounds of (2) and (3) as follows. On the one hand, the upper bound of $c_{k}$ in (3) implies that

$$
\lim \sup c_{k} \leq(1-x)^{2}+x^{2} \lim \sup c_{k} .
$$

On the other hand, the lower bound of $c_{k}$ in (2) implies that

$$
1-x-x \lim \sup c_{k} \leq \liminf c_{k}
$$

Hence, $\lim \sup c_{k}=\lim \inf c_{k}=(1-x) /(1+x)$. This proves Lemma 12 .

Lemma 13 is a consequence of the following trick. Part (i) involves

$$
\frac{k-c}{Z_{1}-c}=1-\frac{Z_{1}-k}{Z_{1}-c}=: 1-v .
$$

By exchangeability, $\mathrm{E}_{k}^{x}(v)$ is $k$ times the expected value of $\left(L_{1}-1\right) /\left(Z_{1}-c\right)$, where $L_{1}$ denotes the number of descendants of the first individual in the initial population. The event $\left\{L_{1}-1 \neq 0\right\}$ is equivalent to $\left\{L_{1}=2\right\}$ and has probability $x$. Thus, for every $k \geq 2$,

$$
(k-c) \mathrm{E}_{k}^{x}\left(\frac{1}{Z_{1}-c}\right)=1-k x \mathrm{E}_{k-1}^{x}\left(\frac{1}{Z_{1}+2-c}\right) .
$$

With the convention that $Z_{1}=0 \mathrm{P}_{0}^{x}$-almost surely, this relation holds for $k=1$ as well. This translates inequality (i) or (i') into inequality (ii) or (ii'). The translation of inequality (ii) or (ii') into inequality (iii) or (iii') uses the same technique, starting from $1 /\left(Z_{1}+2-c\right)$. This concludes the proof of Proposition 16.

\subsection{Algorithm}

The following algorithm yields approximate values of $B_{k}$ on $\mathbb{L}^{*}$, to any prescribed accuracy.

- Fix $n \geq 1$.

- For every $k \geq n+1$ and $x$, let $B_{k, n}^{0}(x):=1 / k$ and $B_{k, n}^{1}(x):=1 /(k-1)$.

- Find the unique sequence $\left(B_{k, n}^{1}\right)_{k \leq n}$ that solves the system of equations (1) for $k \leq n$ when every $B_{k}(s(x)), k \geq n+1$, is replaced with $B_{k, n}^{1}(s(x))$, that is, with the value $1 /(k-1)$.

- Likewise, find the unique sequence $\left(B_{k, n}^{0}(x)\right)_{k \leq n}$ that solves the system of equations (1) for $k \leq n$ when every $B_{k}(s(x)), k \geq n+1$, is replaced with $B_{k, n}^{1}(x)$, that is, with the value $1 / k$.

- Then, for every $k \geq 1$ and every $x$,

$$
B_{k, n}^{0}(x) \leq B_{k}(x) \leq B_{k, n}^{1}(x) \leq(1+1 / n) B_{k, n}^{0}(x) .
$$




\subsection{Comments on the algorithm}

Neither $\left(B_{k, n}^{0}\right)_{k \geq 1}$ nor $\left(B_{k, n}^{1}\right)_{k \geq 1}$ solves the full system of equations (1). For any fixed values of $k$ and $x,\left(B_{k, n}^{0}(x)\right)_{n \geq 1}$ is a nondecreasing sequence that converges to $B_{k}(x)$ as $n \rightarrow \infty$. Likewise, $\left(B_{k, n}^{1}(x)\right)_{n \geq 1}$ is a nonincreasing sequence that converges to $B_{k}(x)$ as $n \rightarrow \infty$. Increasing values of $n$ yield more and more accurate approximations of each $B_{k}(x)$, and the relative error is of order at most $1 / n$.

In the Bernoulli case, we can use initial values better than $B_{k, n}^{0}(x)$ and $B_{k, n}^{1}(x)$, namely, for every $k \geq n+1$ and $x$,

$$
b_{k, n}^{0}(x):=\frac{1}{k-\alpha_{1}(x)}, \quad b_{k, n}^{1}(x):=\frac{1}{k-\alpha_{2}(x)} .
$$

The relative error, which was at most $1+1 / n$ in the first version of the algorithm, is now at most

$$
1+\frac{\alpha_{2}(x)-\alpha_{1}(x)}{n+1-\alpha_{2}(x)} \leq 1+\frac{3-2 \sqrt{2}}{n+x} .
$$

Numerically, this is at most $1+0.172 / n$, for every $x$.

\subsection{Simulations in the homogeneous case}

Using the algorithm above with $n:=1000$ prompts the following refinements. Define $B(x):=B_{1}(x) x=B_{2}(x)(1+x)$. Simulations show that $B$ decreases on $\left(0, x_{*}\right)$ from $B\left(0^{+}\right)=1$ to $B\left(x_{*}\right)=: B_{*}$ and increases on $\left(x_{*}, 1\right]$ from $B_{*}$ to $B(1)=1$, where

$$
x_{*}=0.38 \pm 0.01, \quad B_{*}=0.9274 \pm 0.0002 .
$$

This implies that, for every positive $x$,

$$
B_{*} / x \leq B_{1}(x) \leq 1 / x .
$$

Simulations show that $B_{2}$ and, hence, $B_{1}$ decrease on $(0,1]$.

\section{Size-biased offspring}

When computing harmonic means, it may prove convenient to use size-biased distributions, defined as follows. Assume that $L$ and $L_{i}$ are independent, identically distributed positive, integrable random variables and that $L^{\prime}$ is an independent, size-biased copy of $L$, that is, for every $t$ in $[0,1]$,

$$
\mathrm{E}\left(t^{L^{\prime}}\right):=\mathrm{E}\left(L t^{L}\right) / \mathrm{E}(L)
$$

Then, for any nonnegative integer $k$,

$$
\mathrm{E}(L) \mathrm{E}\left(\frac{1}{L_{1}+\cdots+L_{k}+L^{\prime}}\right)=\frac{1}{k+1} .
$$

For more details on this and many other relations between size-biased distributions and branching mechanisms, see Chapter 10 of Lyons and Peres (2006).

Can we use this in our branching setting? Assume first that $1 \leq L \leq c+1$ almost surely, for a given integer $c$. Since $L^{\prime} \leq c+1 \leq L_{k+1}+\cdots+L_{k+c+1}$ almost surely, this implies that

$$
\mathrm{E}(L) \mathrm{E}_{k}\left(\frac{1}{Z_{1}}\right) \leq \frac{1}{k-c}
$$


for every $k \geq c+1$. More generally, the inequality above holds if $\mathrm{E}\left(t^{L^{\prime}}\right) \geq \mathrm{E}\left(t^{L}\right)^{c+1}$ for every $t$ in $[0,1]$ and a given positive real number $c$.

In our setting, this line of reasoning suffers from two drawbacks. First, it appears that to be able to iterate this inequality over $n$ generations, we must assume that $k \geq n c$. Second, the inequality $\mathrm{E}\left(t^{L^{\prime}}\right) \geq \mathrm{E}\left(t^{L}\right)^{c+1}$ implies that $c \geq A(\xi)$, where $\xi$ denotes the law of $L$ (the proof of this is easy and, thus, omitted). In other words, $k \geq c$ implies that $\xi$ belongs to $\mathcal{A}_{c}$.

\section{The case $k \leq c$}

This section is a brief description of the behaviour of $\mathrm{E}_{k}\left(1 / Z_{n}\right)$ when the hypotheses of Theorem 2 are false. Consider, for the sake of simplicity, a homogeneous branching process and let

$$
p_{i}:=\xi(i)=\mathrm{P}(L=i)=\mathrm{P}\left(Z_{1}=i \mid Z_{0}=1\right) .
$$

Our first remark is that, for every $n \geq 0$ and $k \geq 1$,

$$
\mathrm{E}_{k}\left(1 / Z_{n}\right) \geq r_{k}^{n} / k, \quad r_{k}:=\max \left\{p_{1}^{k}, 1 / \mu\right\}
$$

The bound $1 / \mu$ is due to the convexity. The bound $p_{1}^{k}$ is due to the fact that the probability of the event $\left\{Z_{n}=k\right\}$ is $p_{1}^{k}$.

The parameters $\mu$ and $p_{1}$, through $r_{k}$, indeed describe the asymptotics of $\mathrm{E}_{k}\left(1 / Z_{n}\right)$, as follows. For the sake of simplicity, we exclude the degenerate case $p_{1}^{k} \mu=1$, in which polynomial corrections appear. For every $k \geq 1$, there exists a finite, positive real number $h_{k}$ such that

$$
\lim _{n \rightarrow \infty} \frac{\mathrm{E}_{k}\left(1 / Z_{n}\right)}{r_{k}^{n}}=h_{k} .
$$

In the Bernoulli case, $p_{1}<1 / \mu$ and, hence, $r_{k}=1 / \mu$ for every $k \geq 1$, and the $r_{k}=p_{1}^{k}$ regime is nonexistent.

The limits $h_{k}$ satisfy the following relations. Assume, for instance, that we wish to compute $h_{1}$ and that $\mu p_{1}>1$, whence $r_{1}=p_{1}$. By conditioning on the value of $Z_{1}$, we obtain a relation between $\mathrm{E}_{1}\left(1 / Z_{n+1}\right)$ and the sequence $\left(\mathrm{E}_{k}\left(1 / Z_{n}\right)\right)_{k \geq 1}$. Letting $n$ go to infinity yields

$$
h_{1}=1+\sum_{k \geq 2} \frac{H_{k} p_{k}}{p_{1}}, \quad H_{k}:=\sum_{n \geq 0} \frac{\mathrm{E}_{k}\left(1 / Z_{n}\right)}{p_{1}^{n}} .
$$

The term $\mathrm{E}_{2}\left(1 / Z_{n}\right) / p_{1}^{n}$ behaves like $\left(r_{2} / p_{1}\right)^{n}$, that is, like $1 /\left(\mu p_{1}\right)^{n}$ if $\mu p_{1}^{2}>1$ and like $p_{1}^{n}$ if $\mu p_{1}^{2}<1$. Since both quantities are summable, $H_{2}$ is finite. Since $H_{k} \leq H_{2}$ for every $k \geq 2$, $h_{1}$ is finite as well.

When $\mu p_{1}<1, r_{k}=1 / \mu$ for every $k \geq 1$ and the same reasoning as above yields

$$
h_{1}=\mu \sum_{k \geq 1} p_{k} h_{k}
$$

Since $\mu p_{1}<1$, this gives $h_{1}$ as a linear combination of the $h_{k}, k \geq 2$. In turn, for every $k \geq 2$, a one-step recursion similar to the one we used before shows that $h_{k}$ can be written

$$
h_{k}=\mu \sum_{i \geq k} h_{i} \xi^{* k}(i)
$$


Since the coefficient of $h_{k}$ on the right-hand side is $\mu p_{1}^{k}<1$, this relation implies that $h_{k}$ is a linear combination of the sequence $\left(h_{i}\right)_{i \geq k+1}$, and that this linear combination has nonnegative coefficients. Since $h_{i} \leq h_{2}$ for every $i \geq k+1$, the series converges. However, it does not seem easy to recover information about the coefficients $h_{1}$ or $h_{k}, k \geq 2$, from these relations.

\section{References}

Bingham, N. H. (1988). On the limit of a supercritical branching process. In A Celebration of Applied Probability (J. Appl. Prob. Spec. Vol. 25A), ed. J. Gani, Applied Probability Trust, Sheffield, pp. 215-228.

Dubuc, S. M. (1971). La densité de la loi-limite d'un processus en cascade expansif. Z. Wahrscheinlichkeitsth. 19, 281-290.

HARRIS, T. E. (1948). Branching processes. Ann. Math. Statist. 19, 474-494.

Lyons, R. And Peres, Y. (2006). Probability on Trees and Networks. Unpublished manuscript. Available at http://mypage.iu.edu/ rdlyons/prbtree/prbtree.html.

Ney, P. E. AND Vidyashankar, A. N. (2003). Harmonic moments and large deviation rates for supercritical branching processes. Ann. Appl. Prob. 13, 475-489.

PIau, D. (2001). Processus de branchement en champ moyen et réaction PCR. Adv. Appl. Prob. 33, $391-403$.

PIAU, D. (2004). Immortal branching Markov processes: averaging properties and PCR applications. Ann. Prob. 32, 337-364.

PiaU, D. (2005). Confidence intervals for nonhomogeneous branching processes and polymerase chain reactions. Ann. Prob. 33, 674-702.

Sun, F. (1995). The polymerase chain reaction and branching processes. J. Comput. Biol. 2, 63-86.

Widder, D. V. (1941). The Laplace Transform (Princeton Math. Ser. 6). Princeton University Press. 